\title{
A representação de Kraus para a dinâmica de sistemas quânticos abertos
}

The Kraus representation for the dynamics of open quantum systems

\author{
Jonas Maziero*1,2 \\ ${ }^{1}$ Departamento de Física, Centro de Ciências Naturais e Exatas, Universidade Federal de Santa Maria, \\ Santa Maria, RS, Brazil \\ ${ }^{2}$ Instituto de Física, Facultad de Ingeniería, Universidad de la República, Montevideo, Uruguay
}

Recebido em 2 de dezembro de 2015. Revisado em 11 de janeiro de 2016. Aceito em 10 de fevereiro de 2016

A necessidade e utilidade de se considerar a interação com o ambiente quando da descrição da evolução temporal de sistemas quânticos vem sendo reconhecida nos mais variados ramos da física e de outras ciências. A representação de Kraus é uma forma geral e sucinta para descrever a dinâmica de sistemas quânticos abertos em muitas situações físicas relevantes. Neste artigo, nos abstendo da generalidade do formalismo de operações quânticas e evitando assim as complicações associadas, mostramos de forma simples como obter tal representação usando basicamente a evolução unitária do sistema fechado (sistema mais ambiente) e a função traço parcial. O exemplo de um átomo de dois níveis interagindo com o vácuo do campo eletromagnético é considerado para ilustrar a aplicação desse formalismo, que por fim é utilizado para estudar a evolução temporal da coerência quântica do átomo.

Palavras-chave: mecânica quântica, sistemas abertos, representação de Kraus.

The necessity and utility of considering the interaction of a quantum system with its environment when describing its time evolution have been recognized in several branches of physics and of other sciences. The Kraus' representation is a general and succinct approach to describe such open system dynamics in a wide range of relevant physical scenarios. In this article, by abdicating from the generality of the formalism of quantum operations and with this avoiding its associated complications, we show in a simple manner how we can obtain the Kraus' representation using basically the closed system (system plus environment) unitary dynamics and the partial trace function. The example of a two-level atom interacting with the vacuum of the electromagnetic field is regarded for the sake of instantiating this formalism, which is then applied to study the time evolution of the atom's quantum coherence.

Keywords: quantum mechanics, open systems, Kraus' representation.

\section{Introdução}

A mecânica quântica (MQ) é um dos grandes triunfos obtidos na construção do conhecimento humano e é a base sobre a qual se desenvolveu boa parte das tecnologias atuais [1]. Hoje em dia, informação possui um papel fundamental em nossas vidas e por isso dizemos que estamos na era da informação. Ainda nesse século entraremos em outra etapa da nossa história, a era da informação quântica [2,3]. Testemunharemos a realização de experimentos controlando propriedades físicas tais como coerência e

*Endereço de correspondência: jonas.maziero@ufsm.br correlações quânticas de forma que jamais foi cogitada pelos precursores da MQ (veja e.g. as Refs. [4], p. 70, e [5]), nem pela maioria de nós hoje em dia. Mesclando essa habilidade com a engenhosidade instigada pela corrida tecnológica, lograremos construir máquinas e dispositivos que terão um impacto inimaginável na maneira como vivemos e interagimos.

Embora haja muito ainda por ser feito, já conseguimos obter avanços importantes em várias subáreas do que chamamos atualmente de ciência da informação quântica (CIQ), mas que na verdade engloba muitos ramos da ciência. Investimentos substanciais estão sendo feitos, por exemplo, para 
construir computadores e simuladores quânticos que poderão resolver alguns problemas matemáticos e simular o comportamento de sistemas complexos de maneira muito mais eficiente que os computadores convencionais, clássicos, o fazem [6,7]. Este é só um de diversos exemplos de iniciativas promissoras em CIQ, que vão desde a possibilidade de comunicação absolutamente segura [8] e de medidas mais precisas em metrologia [9, 10, passando pela termodinâmica quântica de sistemas "pequenos" 11 e pelo entendimento de e inspiração em sistemas biológicos 12 e indo até a ramos tão abrangentes quanto a inteligência artificial 13 16] .

Tendo essa perspectiva em vistas, devemos reconhecer a importância de prepararmos e educarmos nossas crianças e jovens para que esse "novo mundo" seja mais natural (menos estranho) para eles do que é para nós. Por isso, iniciativas objetivando facilitar o entendimento da MQ são necessárias $17-22$. A motivação para este artigo vem da observação de que na maioria dos livros texto sobre MQ, tipicamente se assume, implicitamente, que o sistema físico de interesse é fechado, ou seja, que não interage como o seu ambiente e que sua dinâmica é unitária. Aqui esperamos contribuir para o ensino de MQ apresentando de maneira simples a representação de Kraus para a dinâmica quântica de sistemas abertos, o que é o caso para a maioria dos sistemas físicos reais.

Uma maneira interessante para iniciar o aprendizado mais aprofundado de MQ é, depois de ter obtido uma boa base de álgebra linear e de ter feito leituras sobre a fenomenologia da MQ, estudar seus postulados 23 27. Um apresentação didática dos postulados dos estados e das medidas pode ser encontrada na Ref. 27]. Aqui focaremos no postulado da dinâmica. Antes de apresentá-lo, no entanto, lembremos que o estado de um sistema físico é descrito, de forma mais geral, por um operador densidade, que é um operador linear, positivo semi-definido e que possui traço igual a um ${ }^{1}$.

O postulado da dinâmica diz que, se $\hat{H}$ é o operador hamiltoniano (energia) de um sistema isolado no instante de tempo $t$, seu estado evolui no tempo

\footnotetext{
${ }^{1}$ Um operador $\hat{O}$ é linear se $\hat{O}\left(\sum_{j} c_{j}\left|\psi_{j}\right\rangle\right)=\sum_{j} c_{j} \hat{O}\left(\left|\psi_{j}\right\rangle\right)$, onde $c_{j} \in \mathbb{C}$ e $\left\{\left|\psi_{j}\right\rangle\right\}$ é um conjunto qualquer de vetores do espaço de Hilbert $\mathcal{H}$ 27. Esse operador é positivo semidefinido (notação $\hat{O} \geq 0$, onde $\hat{0}$ é o operador nulo) se $\langle\psi|\hat{O}| \psi\rangle \geq 0$ para todo vetor $|\psi\rangle \in \mathcal{H}$. O traço de $\hat{O}$ é definido como: $\operatorname{Tr}(\hat{O})=\sum_{j=1}^{d}\left\langle\xi_{j}|\hat{O}| \xi_{j}\right\rangle$, com $d$ sendo a dimensão e $\left\{\left|\xi_{j}\right\rangle\right\}$ uma base de $\mathcal{H}$.
}

através de uma transformação unitária, ou seja,

$$
\hat{\rho}_{t}=\hat{U} \hat{\rho} \hat{U}^{\dagger}
$$

em que $\hat{\rho}$ é o estado inicial (em $t=0$ ) do sistema e o operador de evolução temporal é obtido a partir da equação de Schrödinger:

$$
i \hbar \partial_{t} \hat{U}=\hat{H} \hat{U}
$$

em que $\hbar$ é a constante de Planck e, neste artigo, usaremos a notação $\partial_{t} \equiv \frac{\partial}{\partial t}$. Não é difícil verificar que as Eqs. (1) e (2) são equivalentes à equação de von Neumann 2

$$
i \hbar \partial_{t} \hat{\rho}_{t}=\left[\hat{H}, \hat{\rho}_{t}\right]
$$

Vale mencionar que, por suas estruturas algébricas, as Eqs. (1) e (3) são chamadas, respectivamente, de versões discreta ${ }^{3}$ e contínud ${ }^{4}$ da dinâmica quântica de sistemas fechados. Neste artigo trabalharemos com a generalização para sistemas abertos da versão discreta, que é devida a Kraus 28,29] e é bastante útil para descrever a evolução temporal de vários sistemas físicos reais. Para mais informações sobre generalizações da versão contínua, tais como os formalismos de Redfield, das equações mestras e das trajetórias quânticas, veja 30 33 e suas referências.

Quando lidamos com dois (ou mais) graus de liberdade, chamados e.g. de $S$ e $A$, ou seja, quando consideramos sistemas compostos, complementamos o postulado dos estados 27] assumindo que o estado do sistema como um todo é descrito por um operador densidade definido no espaço de Hilbert que é obtido tomando-se o produto tensorial dos espaços individuais, i.e., $\mathcal{H}_{S} \otimes \mathcal{H}_{A}=: \mathcal{H}_{S A}[23$. Neste contexto, frequentemente conhecemos o estado global mas queremos calcular probabilidades relacionadas a somente um dos sub-sistemas. Para isso é conveniente usar a função linear traço parcial, cuja definição é [24]:

$$
\begin{aligned}
\operatorname{Tr}_{A}(|\psi\rangle\langle\phi|\otimes| \xi\rangle\langle\zeta|) & :=|\psi\rangle\langle\phi| \otimes \operatorname{Tr}_{A}(|\xi\rangle\langle\zeta|) \\
& =\langle\zeta \mid \xi\rangle|\psi\rangle\langle\phi|,
\end{aligned}
$$

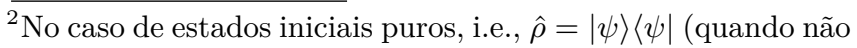
existe incerteza clássica sobre a preparação do sistema [27]), temos $\left|\psi_{t}\right\rangle=\hat{U}|\psi\rangle$, que, junto com a Eq. (1), é equivalente à equação de Schrödinger para o estado: $i \hbar \partial_{t}\left|\psi_{t}\right\rangle=\hat{H}\left|\psi_{t}\right\rangle$.

${ }^{3}$ Pois, uma vez que $\hat{U}$ é conhecido, essa equação é uma mapa discreto $\hat{\rho} \mapsto \hat{\rho}_{t}$.

${ }^{4}$ Pois essa equação é uma equação diferencial que envolve variações de $\hat{\rho}$ em intervalos de tempo infinitesimais em torno do instante de tempo $t$. 
em que $|\psi\rangle,|\phi\rangle \in \mathcal{H}_{S}$ e $|\xi\rangle,|\zeta\rangle \in \mathcal{H}_{A}$ e tomamos o traço sobre $\mathcal{H}_{A}$. Assim, por exemplo, se o estado do sistema bipartido $S A$ é $\hat{\rho}$, o estado do sistema $S$ pode ser obtido usando-se o traço parcial:

$$
\hat{\rho}^{S}=\operatorname{Tr}_{A}(\hat{\rho}) .
$$

A continuação deste artigo está organizada da seguinte forma. Começaremos, na Sec. 2, obtendo a representação de Kraus para a dinâmica de um sistema quântico aberto. Na sequência, na Sec. 3 . forneceremos um exemplo de aplicação dessa representação na descrição da evolução temporal de um átomo de dois níveis, e de sua coerência quântica, quando esse interage com o campo eletromagnético inicialmente no estado de vácuo. Deixamos para o Apêndice as provas das várias propriedades que são exigidas (ou esperadas) para os operadores e a dinâmica de Kraus.

\section{A representação de Kraus a partir da dinâmica unitária}

Nesta seção obteremos, de maneira simples e sem ambiguidades, a representação de Kraus para o estado evoluído de um sistema quântico discreto. Tentamos simplificar ao máximo a notação para que o material seja acessível a todos os que possuem um conhecimento básico do formalismo matemático da MQ de sistemas fechados. Para evitar as complicações relacionadas à questão da possível não positividade completa da dinâmica quântica gerada a partir de estados iniciais correlacionados [34 40], vamos considerar que sistema e ambiente estão inicialmente em um estado produto:

$$
\hat{\rho}=\hat{\rho}^{S} \otimes\left|E_{0}\right\rangle\left\langle E_{0}\right| .
$$

O ambiente propriamente dito, ou seja, a parte do universo que pode interagir efetivamente com o sistema $S$ em uma escala de tempo relevante, poderia estar em um estado misto qualquer. No entanto, para facilitar as demonstrações, vamos utilizar uma purificação deste estado [24 26]; veja a Fig. 1.

O primeiro passo para a obtenção da representação de Kraus é considerar o sistema $S$ mais seu ambiente $A$ como sendo um sistema fechado. Assim, o estado desse sistema composto será evoluído no tempo através de uma transformação unitária, como mostrado na Eq. (1), com $\hat{U}$ obtido da equação de Schrödinger, Eq. (2), com

$$
\hat{H}=\hat{H}_{S} \otimes \mathbb{I}_{A}+\mathbb{I}_{S} \otimes \hat{H}_{A}+g \hat{H}_{\text {int }},
$$

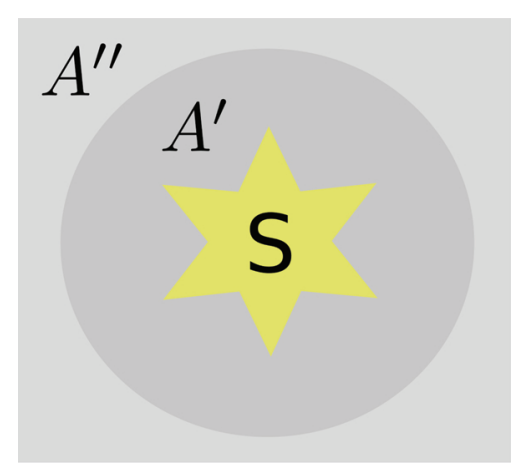

Figura 1: $\mathrm{O}$ sistema $S$, preparado no estado $\hat{\rho}^{S}$, pode ser influenciado efetivamente pelo ambiente $A^{\prime}$. Se a decomposição espectral do estado inicial de $A^{\prime}$ é $\hat{\rho}_{A^{\prime}}=$ $\sum_{j} a_{j}^{\prime}\left|a_{j}^{\prime}\right\rangle\left\langle a_{j}^{\prime}\right|$, podemos utilizar a decomposição de Schmidt 24] e outro ambiente $A^{\prime \prime}$ para purificar esse estado: $\hat{\rho}_{A^{\prime}}=\operatorname{Tr}_{A^{\prime \prime}}\left(\left|E_{0}\right\rangle\left\langle E_{0}\right|\right)$, com $\left|E_{0}\right\rangle:=\sum_{j} \sqrt{a_{j}^{\prime}}\left|a_{j}^{\prime}\right\rangle \otimes\left|a_{j}^{\prime \prime}\right\rangle \in$ $\mathcal{H}_{A^{\prime}} \otimes \mathcal{H}_{A^{\prime \prime}} \equiv \mathcal{H}_{A^{\prime} A^{\prime \prime}}=: \mathcal{H}_{A}$ e $\left\{\left|a_{j}^{\prime \prime}\right\rangle\right\}$ é uma base ortonormal de $\mathcal{H}_{A^{\prime \prime}}$.

onde $\mathbb{I}_{S(A)}$ é o operador identidade no espaço vetorial correspondente, $\hat{H}_{S(A)}$ é o chamado Hamiltoniano livre do sistema (ambiente) e $g$ é um parâmetro real que determina o "quão forte" é a interação entre o sistema e seu ambiente, cuja forma é descrita pelo Hamiltoniano de interação $\hat{H}_{\text {int }}$. Se $g=0$ sistema e ambiente evoluem de forma independente, e continuam descorrelacionados. Por outro lado, se $g \neq 0$ correlações são geradas entre sistema e ambiente [41, 42], o que pode influenciar as característcas quânticas de $S$ causando, por exemplo, perda de coerência quântica em um processo conhecido como decoerência [43, 44].

O leitor certamente já verificou que operadores unitários preservam o produto escalar entre vetores do espaço no qual atuam e que o efeito dessas transformações é simplesmente o de realizar uma mudança de base, ou de representação. Então, se consideramos duas bases quaisquer de vetores ortonormais do espaço de Hilbert para o sistema composto,

$$
\left\{\left|\Phi_{j}\right\rangle\right\},\left\{\left|\Psi_{j}\right\rangle\right\} \in \mathcal{H}_{S} \otimes \mathcal{H}_{A},
$$

com $j=0, \cdots, d_{S} d_{A}-1$, onde $d_{S(A)}=\operatorname{dim}\left(\mathcal{H}_{S(A)}\right)$ é a dimensão do espaço de estados para o sistema (ambiente), podemos escrever:

$$
\hat{U}=\sum_{j}\left|\Phi_{j}\right\rangle\left\langle\Psi_{j}\right|
$$

As bases que aparecem na última equação definem a ação do operador unitário e são completamente 
gerais, podendo não ser um simples produto tensorial de bases de $\mathcal{H}_{S}$ e de $\mathcal{H}_{A}$, fato que dependerá da forma do Hamiltoniano de interação. Será útil, para alcançarmos nosso objetivo aqui, escrever os vetores dessas duas bases gerais em termos de uma base produto:

$$
\left\{\left|S_{k}\right\rangle \otimes\left|E_{l}\right\rangle=:\left|S_{k} E_{l}\right\rangle\right\}
$$

onde $k=0, \cdots, d_{S}-1$ and $l=0, \cdots, d_{A}-1$. A relação de completeza em $\mathcal{H}_{S A}$,

$$
\mathbb{I}=\sum_{k l}\left|S_{k} E_{l}\right\rangle\left\langle S_{k} E_{l}\right|
$$

é então utilizada para escrever

$$
\begin{aligned}
\left|\Phi_{j}\right\rangle & =\mathbb{I}\left|\Phi_{j}\right\rangle \\
& =\sum_{k l}\left|S_{k} E_{l}\right\rangle\left\langle S_{k} E_{l}|| \Phi_{j}\right\rangle \\
& :=\sum_{k l} f_{k l}^{(j)}\left|S_{k} E_{l}\right\rangle,
\end{aligned}
$$

onde definimos os coeficientes complexos

$$
f_{k l}^{(j)}:=\left\langle S_{k} E_{l} \mid \Phi_{j}\right\rangle
$$

Analogamente

$$
\left|\Psi_{j}\right\rangle=\sum_{k l} g_{k l}^{(j)}\left|S_{k} E_{l}\right\rangle
$$

com os coeficientes

$$
g_{k l}^{(j)}:=\left\langle S_{k} E_{l} \mid \Psi_{j}\right\rangle
$$

Assim o operador unitário toma a forma:

$$
\begin{aligned}
\hat{U} & =\sum_{j} \sum_{k l} f_{k l}^{(j)}\left|S_{k} E_{l}\right\rangle \sum_{m n} g_{m n}^{(j) *}\left\langle S_{m} E_{n}\right| \\
& =\sum_{j k l m n} f_{k l}^{(j)} g_{m n}^{(j) *}\left|S_{k} E_{l}\right\rangle\left\langle S_{m} E_{n}\right|,
\end{aligned}
$$

em que usamos o fato de que $(c|\xi\rangle)^{\dagger}=c^{*}\langle\xi|$ para qualquer $c \in \mathbb{C}$, com $c^{*}$ sendo o complexo conjugado de $c$.

Usando o operador unitário escrito em termos da base produto, aplicado como mostrado na Eq. (1), e a função traço parcial, o estado evoluído do sistema $S$,

$$
\hat{\rho}_{t}^{S}=\operatorname{Tr}_{A}\left(\hat{\rho}_{t}\right)=\operatorname{Tr}_{A}\left(\hat{U} \hat{\rho} \hat{U}^{\dagger}\right),
$$

é obtido como segue:

$$
\begin{aligned}
\hat{\rho}_{t}^{S} & =\operatorname{Tr}_{A} \sum_{j k l m n} f_{k l}^{(j)} g_{m n}^{(j) *}\left|S_{k} E_{l}\right\rangle\left\langle S_{m} E_{n}\left|\left(\hat{\rho}^{S} \otimes\left|E_{0}\right\rangle\left\langle E_{0}\right|\right) \sum_{o p q r s} f_{p q}^{(o) *} g_{r s}^{(o)}\right| S_{r} E_{s}\right\rangle\left\langle S_{p} E_{q}\right| \\
& =\operatorname{Tr}_{A} \sum_{j k l m n o p q r s} f_{k l}^{(j)} g_{m n}^{(j) *} f_{p q}^{(o) *} g_{r s}^{(o)}\left|S_{k} E_{l}\right\rangle\left\langle S_{m} E_{n}\left|\left(\hat{\rho}^{S} \otimes\left|E_{0}\right\rangle\left\langle E_{0}\right|\right)\right| S_{r} E_{s}\right\rangle\left\langle S_{p} E_{q}\right| \\
& =\operatorname{Tr}_{A} \sum_{j k l m n o p q r s} f_{k l}^{(j)} g_{m n}^{(j) *} f_{p q}^{(o) *} g_{r s}^{(o)}\left|S_{k}\right\rangle\left\langle S_{m}\left|\hat{\rho}^{S}\right| S_{r}\right\rangle\left\langle S_{p}|\otimes| E_{l}\right\rangle \underbrace{\left\langle E_{n} \mid E_{0}\right\rangle\left\langle E_{0} \mid E_{s}\right\rangle}_{=\delta_{n 0}}\left\langle E_{q}\right| \\
& =\sum_{j k l m o p q r} f_{k l}^{(j)} g_{m 0}^{(j) *} f_{p q}^{(o) *} g_{r 0}^{(o)}\left|S_{k}\right\rangle\left\langle S_{m}\left|\hat{\rho}^{S}\right| S_{r}\right\rangle\left\langle S_{p}\right| \underbrace{\operatorname{Tr}_{A}\left(\left|E_{l}\right\rangle\left\langle E_{q}\right|\right)}_{=\left\langle E_{q} \mid E_{l}\right\rangle=\delta_{q l}} \\
& =\sum_{j k l m o p r} f_{k l}^{(j)} g_{m 0}^{(j) *} f_{p l}^{(o) *} g_{r 0}^{(o)}\left|S_{k}\right\rangle\left\langle S_{m}\left|\hat{\rho}^{S}\right| S_{r}\right\rangle\left\langle S_{p}\right| \\
& =\sum_{j k l m o p r}\left\langle S_{k} E_{l} \mid \Phi_{j}\right\rangle\left\langle\Psi_{j} \mid S_{m} E_{0}\right\rangle\left\langle\Phi_{o} \mid S_{p} E_{l}\right\rangle\left\langle S_{r} E_{0} \mid \Psi_{o}\right\rangle\left|S_{k}\right\rangle\left\langle S_{m}\left|\hat{\rho}^{S}\right| S_{r}\right\rangle\left\langle S_{p}\right| \\
& =\sum_{j k l m o p r}\left\langle S_{k} E_{l} \mid \Phi_{j}\right\rangle\left\langle\Psi_{j} \mid S_{m} E_{0}\right\rangle\left|S_{k}\right\rangle\left\langle S_{m}\left|\hat{\rho}^{S}\right| S_{r}\right\rangle\left\langle S_{p}\right|\left\langle S_{r} E_{0} \mid \Psi_{o}\right\rangle\left\langle\Phi_{o} \mid S_{p} E_{l}\right\rangle \\
& =\sum_{k l m p r}\left\langle S_{k} E_{l}\left|\left(\sum_{j}\left|\Phi_{j}\right\rangle\left\langle\Psi_{j}\right|\right)\right| S_{m} E_{0}\right\rangle\left|S_{k}\right\rangle\left\langle S_{m}\left|\hat{\rho}^{S}\right| S_{r}\right\rangle\left\langle S_{p}\right|\left\langle S_{r} E_{0}\left|\left(\sum_{o}\left|\Psi_{o}\right\rangle\left\langle\Phi_{o}\right|\right)\right| S_{p} E_{l}\right\rangle \\
& =\sum_{k l m p r}\left\langle S_{k} E_{l}|\hat{U}| S_{m} E_{0}\right\rangle\left|S_{k}\right\rangle\left\langle S_{m}\left|\hat{\rho}^{S}\right| S_{r}\right\rangle\left\langle S_{p}\right|\left\langle S_{r} E_{0}\left|\hat{U}^{\dagger}\right| S_{p} E_{l}\right\rangle .
\end{aligned}
$$


Acima $\delta_{j k}$ é a função delta de Kronecker. Na passagem da Eq. (20) para a Eq. (21) lembramos que $\left|S_{k} E_{l}\right\rangle=\left|S_{k}\right\rangle \otimes\left|E_{l}\right\rangle$ e usamos o fato de que

$$
(\hat{A} \otimes \hat{B})(\hat{C} \otimes \hat{D})=\hat{A} \hat{C} \otimes \hat{B} \hat{D}
$$

para quaisquer quatro matrizes complexas $\hat{A}, \hat{B}, \hat{C}, \hat{D}$. Para obter 22 de (21) usamos a linearidade do traço parcial. Já a Eq. (24) é obtida a partir de (23) usando as expressões para os coeficientes complexos definidos acima em termos do produto interno entre os vetores base. Rearranjando os termos da Eq. (25) "fazemos aparecer" os operadores unitários de (26), o que leva à Eq. (27).
Prosseguindo definimos os elementos de matriz dos operadores de Kraus na base $\left\{\left|S_{k}\right\rangle\right\}$ em termos dos elementos de matriz de $\hat{U}$ na base produtc ${ }^{5}$.

$$
\left\langle S_{k}\left|\hat{K}_{l}\right| S_{m}\right\rangle:=\left\langle S_{k} E_{l}|\hat{U}| S_{m} E_{0}\right\rangle
$$

Essa relação implica em $\left\langle S_{m}\left|\hat{K}_{l}^{\dagger}\right| S_{k}\right\rangle=\left\langle S_{m} E_{0}\left|\hat{U}^{\dagger}\right| S_{k} E_{l}\right\rangle$ e portanto

$$
\begin{aligned}
\hat{\rho}_{t}^{S}= & \sum_{k l m p r}\left\langle S_{k}\left|\hat{K}_{l}\right| S_{m}\right\rangle\left|S_{k}\right\rangle\left\langle S_{m}\left|\hat{\rho}^{S}\right| S_{r}\right\rangle\left\langle S_{p}\right|\left\langle S_{r}\left|\hat{K}_{l}^{\dagger}\right| S_{p}\right\rangle= \\
& \sum_{k l m p r}\left|S_{k}\right\rangle\left\langle S_{k}\left|\hat{K}_{l}\right| S_{m}\right\rangle\left\langle S_{m}\left|\hat{\rho}^{S}\right| S_{r}\right\rangle\left\langle S_{r}\left|\hat{K}_{l}^{\dagger}\right| S_{p}\right\rangle\left\langle S_{p}\right|= \\
& \sum_{l} \mathbb{I}_{S} \hat{K}_{l} \mathbb{I}_{S} \hat{\rho}^{S} \mathbb{I}_{S} \hat{K}_{l}^{\dagger} \mathbb{I}_{S} .
\end{aligned}
$$

Chegamos assim à representação da soma de operadores de Kraus:

$$
\hat{\rho}_{t}^{S}=\sum_{l} \hat{K}_{l} \hat{\rho}^{S} \hat{K}_{l}^{\dagger}
$$

É bastante comum encontrarmos o operador densidade $\hat{\rho}_{t}^{S}$ escrito como $\$\left[\hat{\rho}^{S}\right]$ (ou com outro símbolo no lugar de $\$$ ). Esse procedimento enfatiza que a operação quântica $\$$ é um mapa (discreto) entre os estados $\hat{\rho}^{S}$ e $\hat{\rho}_{t}^{S}$, o que é denotado por

$$
\$: \hat{\rho}^{S} \mapsto \hat{\rho}_{t}^{S} .
$$

Como $\$$ é um mapa que leva operador em operador ele é, algumas vezes, chamado de super-operador. No Apêndice mostramos que os operadores de Kraus da Eq. (29) estão definidos em $\mathcal{H}_{S}$, que são lineares

\footnotetext{
${ }^{5}$ Queremos chamar a atenção para o fato de não ser raro encontrarmos os operadores de Kraus escritos da seguinte forma (veja por exemplo a Ref. 24 ): $\hat{K}_{l}=\left\langle E_{l}|\hat{U}| E_{0}\right\rangle$. Embora o significado dessa equação seja claro para quem está familiarizado com o assunto, evitamos utilizá-la neste artigo pois, além do produto matricial $\hat{U}\left|E_{0}\right\rangle$ não ser bem definido matematicamente, esse tipo de notação pode confundir sem necessidade aqueles que estudam o tópico pela primeira vez.
}

e que a dinâmica gerada por eles (Eq. (31)) preserva o traço e é positiva. Também discutimos a não unicidade do conjunto de operadores de Kraus que geram a evolução temporal de $S$ e o consequente limite no número de tais operadores necessários para descrevê-la.

\section{3. Átomo de dois níveis interagindo com o vácuo do campo eletromagnético}

Como o propósito de exemplificar a aplicação da representação de Kraus, nesta seção vamos considerar um átomo de dois níveis, e.g. um átomo de Rydberg [45 48] ou sistemas análogos [49 51], cujos estados fundamental e excitado serão denotados, respectivamente, por $\left|S_{0}\right\rangle$ e $\left|S_{1}\right\rangle$. O ambiente desse átomo é o campo eletromagnético, que está inicialmente no estado de vácuo (i.e., com nenhuma excitação), que chamaremos de $\left|E_{0}\right\rangle$. Estados do ambiente contendo $j$ fótons espraiados por seus modos são denotados por $\left|E_{j}\right\rangle$. Sob essas condições um processo de emissão "espontânea" ocorrerá por causa da interação do sistema atômico com as flutuações quânticas do vácuo $[52,53$. Essa dinâmica 
pode ser modelada via o seguinte mapa fenomenológico 24,25]:

$$
\begin{aligned}
& \hat{U}_{C A A}\left|S_{0} E_{0}\right\rangle=\left|S_{0} E_{0}\right\rangle, \\
& \hat{U}_{C A A}\left|S_{1} E_{0}\right\rangle=\sqrt{1-p}\left|S_{1} E_{0}\right\rangle+\sqrt{p}\left|S_{0} E_{1}\right\rangle,
\end{aligned}
$$

que em CIQ é conhecido como canal de amortecimento de amplitude (CAA) 24, 25]. A interpretação desse mapa é simples. Se o átomo está inicialmente no estado fundamental, ele continuará assim pois não há energia alguma para "circular" pelos sistemas. No entando, se o átomo estiver inicialmente no estado excitado, este emitirá, em um processo randômico, um fóton com probabilidade $p$ (i.e., $0 \leq p \leq 1$ ), que é proporcional ao tempo de interação átomo-campo. Como o ambiente é muito "grande", vai demorar também um tempo muito grande, considerado na prática como sendo infinito, para que o fóton seja recapturado pelo átomo. Dessa observação inferimos que o estado assimptótico do átomo será $\left|S_{0}\right\rangle$, independentemente de qual seja o seu estado inicial. A probabilidade $p$ é muitas vezes chamada de tempo parametrizado $33,41,42,54$. Se observarmos a taxa $\gamma$ com que um número muito grande desses átomos, todos independentes um do outro, perdem suas excitações, temos que $p \approx 1-\mathrm{e}^{-\gamma t}[54$. Portando $t=0 \mathrm{~s}$ corresponde a $p=0$, enquanto $p=1$ seria o limite assimptótico $t \rightarrow \infty$.

\subsection{Operadores de Kraus para o CAA}

Nesta sub-seção utilizaremos a relação $\left\langle S_{k}\left|\hat{K}_{l}\right| S_{m}\right\rangle:=\left\langle S_{k} E_{l}\left|\hat{U}_{C A A}\right| S_{m} E_{0}\right\rangle$, com a ação do operador unitário dada pelo canal de amortecimento de amplitude (Eqs. (33)), para obter os operadores de Kraus que geram essa dinâmica quântica:

- $\hat{K}_{0}$ :

$$
\begin{aligned}
\left\langle S_{0}\left|\hat{K}_{0}\right| S_{0}\right\rangle & =\left\langle S_{0} E_{0}\left|\hat{U}_{C A A}\right| S_{0} E_{0}\right\rangle \\
& =\left\langle S_{0} E_{0} \mid S_{0} E_{0}\right\rangle=1 . \\
\left\langle S_{0}\left|\hat{K}_{0}\right| S_{1}\right\rangle= & \left\langle S_{0} E_{0}\left|\hat{U}_{C A A}\right| S_{1} E_{0}\right\rangle \\
= & \left\langleS _ { 0 } E _ { 0 } \left|\left(\sqrt{1-p}\left|S_{1} E_{0}\right\rangle+\sqrt{p}\left|S_{0} E_{1}\right\rangle\right)\right.\right. \\
= & 0 . \\
\left\langle S_{1}\left|\hat{K}_{0}\right| S_{0}\right\rangle= & \left\langle S_{1} E_{0}\left|\hat{U}_{C A A}\right| S_{0} E_{0}\right\rangle \\
= & \left\langle S_{1} E_{0} \mid S_{0} E_{0}\right\rangle=0 . \\
\left\langle S_{1}\left|\hat{K}_{0}\right| S_{1}\right\rangle= & \left\langle S_{1} E_{0}\left|\hat{U}_{C A A}\right| S_{1} E_{0}\right\rangle \\
= & \left\langleS _ { 1 } E _ { 0 } \left|\left(\sqrt{1-p}\left|S_{1} E_{0}\right\rangle+\sqrt{p}\left|S_{0} E_{1}\right\rangle\right)\right.\right. \\
= & \sqrt{1-p .} .
\end{aligned}
$$

i.e., na base $\left\{\left|S_{j}\right\rangle\right\}$

$$
\hat{K}_{0}=\left[\begin{array}{cc}
1 & 0 \\
0 & \sqrt{1-p}
\end{array}\right] .
$$

- $\hat{K}_{1}$ :

$$
\begin{gathered}
\left\langle S_{0}\left|\hat{K}_{1}\right| S_{0}\right\rangle=\left\langle S_{0} E_{1}\left|\hat{U}_{C A A}\right| S_{0} E_{0}\right\rangle \\
=\left\langle S_{0} E_{1} \mid S_{0} E_{0}\right\rangle=0 . \\
\left\langle S_{0}\left|\hat{K}_{1}\right| S_{1}\right\rangle=\left\langle S_{0} E_{1}\left|\hat{U}_{C A A}\right| S_{1} E_{0}\right\rangle \\
=\left\langleS _ { 0 } E _ { 1 } \left|\left(\sqrt{1-p}\left|S_{1} E_{0}\right\rangle+\sqrt{p}\left|S_{0} E_{1}\right\rangle\right)\right.\right. \\
=\sqrt{p} .
\end{gathered}
$$




$$
\begin{aligned}
\left\langle S_{1}\left|\hat{K}_{1}\right| S_{0}\right\rangle & =\left\langle S_{1} E_{1}\left|\hat{U}_{C A A}\right| S_{0} E_{0}\right\rangle \\
& =\left\langle S_{1} E_{1} \mid S_{0} E_{0}\right\rangle=0 . \\
\left\langle S_{1}\left|\hat{K}_{1}\right| S_{1}\right\rangle & =\left\langle S_{1} E_{1}\left|\hat{U}_{C A A}\right| S_{1} E_{0}\right\rangle \\
& =\left\langleS _ { 1 } E _ { 1 } \left|\left(\sqrt{1-p}\left|S_{1} E_{0}\right\rangle+\sqrt{p}\left|S_{0} E_{1}\right\rangle\right)\right.\right. \\
& =0 .
\end{aligned}
$$

i.e., na base $\left\{\left|S_{j}\right\rangle\right\}$

$$
\hat{K}_{1}=\left[\begin{array}{cc}
0 & \sqrt{p} \\
0 & 0
\end{array}\right]
$$

- $\hat{K}_{l \geq 2}=\hat{0}$, com 0 sendo o operador nulo, pois há no máximo um fóton no sistema como um todo e por conseguinte $\left\langle S_{m} E_{l \geq 2}\left|\hat{U}_{C A A}\right| S_{n} E_{0}\right\rangle=0$.

\subsection{Dinâmica da coerência quântica sob o CAA}

Segundo Feynman, a coerência quântica (CQ) é o aspecto mais fundamental da mecânica quântica 55]. A palavra coerência (ou mesmo CQ) se fez presente por vários anos em óptica quântica e em áreas correlatas (veja e.g. 56, 57 e suas referências). Nos últimos dois anos tem-se reconsiderado a CQ do ponto de vista de teoria de recursos [58 65], o que gerou um intensa e produtiva atividade de pesquisa sobre esse tema 66 73.

Aqui, evitando demasiados detalhes, diremos que um sistema físico preparado em um certo estado $\hat{\rho}$ possui CQ em relação a uma dada base $\left\{\left|o_{j}\right\rangle\right\}$ (ou observável $\hat{O}=\sum_{j} o_{j}\left|o_{j}\right\rangle\left\langle o_{j}\right|$, com $o_{j} \in \mathbb{R}$ ) se, quando representado naquela base, possuir elementos de matriz fora da diagonal principal $\left(\left\langle o_{j}|\hat{\rho}| o_{k}\right\rangle\right.$ com $j \neq k$ ) não nulos. Note que isso implica na existência de incerteza quântica em relação a qual resultado será obtido em uma medida de $\hat{O}$ [27]. A soma dos módulos desses elementos de matriz nos fornece um boa medida de CQ 67]:

$$
C(\hat{\rho})=\sum_{j \neq k}\left|\left\langle o_{j}|\hat{\rho}| o_{k}\right\rangle\right| .
$$

Vamos estudar como a dinâmica do sistema considerado nesta seção influencia a sua CQ. Assumiremos que o átomo está inicialmente em um estado qualquer:

$$
\hat{\rho}^{S}=\frac{1}{2}\left(\mathbb{I}_{S}+\vec{r} \cdot \overrightarrow{\hat{\sigma}}\right),
$$

onde $\vec{r}=\left(r_{1}, r_{2}, r_{3}\right)$ é o vetor de Bloch, com $r_{j}=$ $\operatorname{Tr}\left(\hat{\rho}^{S} \hat{\sigma}_{j}\right) \in \mathbb{R}$ sendo as "polarizações" para este estado, e $\overrightarrow{\hat{\sigma}}=\left(\hat{\sigma}_{1}, \hat{\sigma}_{2}, \hat{\sigma}_{3}\right)$ com as matrizes de Pauli, na base $\left\{\left|S_{j}\right\rangle\right\}$, sendo escritas como:

$$
\hat{\sigma}_{1}=\left[\begin{array}{ll}
0 & 1 \\
1 & 0
\end{array}\right], \hat{\sigma}_{2}=\left[\begin{array}{cc}
0 & -i \\
i & 0
\end{array}\right], \hat{\sigma}_{3}=\left[\begin{array}{cc}
1 & 0 \\
0 & -1
\end{array}\right] .
$$

Assim, na base $\left\{\left|S_{j}\right\rangle\right\}$,

$$
\hat{\rho}^{S}=\frac{1}{2}\left[\begin{array}{cc}
1+r_{3} & r_{1}-i r_{2} \\
r_{1}+i r_{2} & 1-r_{3}
\end{array}\right]
$$

e a CQ do estado inicial, em relação a essa base, é dada por:

$$
\begin{aligned}
C\left(\hat{\rho}^{S}\right) & =2^{-1}\left(\left|r_{1}-i r_{2}\right|+\left|r_{1}+i r_{2}\right|\right) \\
& =\sqrt{r_{1}^{2}+r_{2}^{2}}
\end{aligned}
$$

em que usamos $r_{1}, r_{2} \in \mathbb{R}$ e, para $z \in \mathbb{C}$,

$$
|z|=\left|z^{*}\right|=\sqrt{\operatorname{Re}(z)^{2}+\operatorname{Im}(z)^{2}} .
$$

Se aplicarmos as Eqs. (38) e (43) ao estado inicial geral da Eq. 45), o estado evoluído do átomo,

$$
\hat{\rho}_{p}^{S}=\sum_{j=0}^{1} \hat{K}_{j} \hat{\rho}^{S} \hat{K}_{j}^{\dagger},
$$

será

$$
\begin{aligned}
\hat{\rho}_{p}^{S} & =\frac{1}{2}\left(\sum_{j=0}^{1} \hat{K}_{j} \mathbb{I}_{S} \hat{K}_{j}^{\dagger}+\sum_{j=0}^{1} \hat{K}_{j} \vec{r} \cdot \overrightarrow{\hat{\sigma}} \hat{K}_{j}^{\dagger}\right) \\
& =\frac{1}{2}\left(\sum_{j=0}^{1} \hat{K}_{j} \hat{K}_{j}^{\dagger}+\sum_{k=1}^{3} r_{k} \sum_{j=0}^{1} \hat{K}_{j} \hat{\sigma}_{k} \hat{K}_{j}^{\dagger}\right)
\end{aligned}
$$


Podemos verificar que

$$
\begin{aligned}
& \sum_{j=0}^{1} \hat{K}_{j} \hat{K}_{j}^{\dagger}=K_{0} K_{0}^{\dagger}+K_{1} K_{1}^{\dagger} \\
& =\left[\begin{array}{cc}
1 & 0 \\
0 & \sqrt{1-p}
\end{array}\right]\left[\begin{array}{cc}
1 & 0 \\
0 & \sqrt{1-p}
\end{array}\right]+\left[\begin{array}{cc}
0 & \sqrt{p} \\
0 & 0
\end{array}\right]\left[\begin{array}{cc}
0 & 0 \\
\sqrt{p} & 0
\end{array}\right] \\
& =\left[\begin{array}{cc}
1 & 0 \\
0 & (1-p)
\end{array}\right]+\left[\begin{array}{ll}
p & 0 \\
0 & 0
\end{array}\right]=\left[\begin{array}{cc}
1 & 0 \\
0 & 1
\end{array}\right]+p\left[\begin{array}{cc}
1 & 0 \\
0 & -1
\end{array}\right] \\
& =\mathbb{I}_{S}+p \hat{\sigma}_{3}
\end{aligned}
$$

e

$$
\begin{aligned}
& \sum_{j=0}^{1} \hat{K}_{j} \hat{\sigma}_{1} \hat{K}_{j}^{\dagger}=\hat{K}_{0} \hat{\sigma}_{1} \hat{K}_{0}^{\dagger}+\hat{K}_{1} \hat{\sigma}_{1} \hat{K}_{1}^{\dagger} \\
& =\left[\begin{array}{cc}
1 & 0 \\
0 & \sqrt{1-p}
\end{array}\right]\left[\begin{array}{ll}
0 & 1 \\
1 & 0
\end{array}\right]\left[\begin{array}{cc}
1 & 0 \\
0 & \sqrt{1-p}
\end{array}\right] \\
& +\left[\begin{array}{cc}
0 & \sqrt{p} \\
0 & 0
\end{array}\right]\left[\begin{array}{ll}
0 & 1 \\
1 & 0
\end{array}\right]\left[\begin{array}{cc}
0 & 0 \\
\sqrt{p} & 0
\end{array}\right] \\
& =\left[\begin{array}{cc}
0 & 1 \\
\sqrt{1-p} & 0
\end{array}\right]\left[\begin{array}{cc}
1 & 0 \\
0 & \sqrt{1-p}
\end{array}\right]+\left[\begin{array}{cc}
\sqrt{p} & 0 \\
0 & 0
\end{array}\right]\left[\begin{array}{cc}
0 & 0 \\
\sqrt{p} & 0
\end{array}\right] \\
& =\left[\begin{array}{cc}
0 & \sqrt{1-p} \\
\sqrt{1-p} & 0
\end{array}\right]+\left[\begin{array}{ll}
0 & 0 \\
0 & 0
\end{array}\right] \\
& =\sqrt{1-p}\left[\begin{array}{ll}
0 & 1 \\
1 & 0
\end{array}\right] \\
& =\hat{\sigma}_{1} \sqrt{1-p} .
\end{aligned}
$$

Da mesma maneira podemos obter a relação geral (para $k=1,2,3$ ):

$$
\sum_{j=0}^{1} \hat{K}_{j} \hat{\sigma}_{k} \hat{K}_{j}^{\dagger}=(\sqrt{1-p})^{\left(1+\delta_{3 k}\right)} \hat{\sigma}_{k} .
$$

Assim o estado evoluído do átomo toma a seguinte forma:

$$
\begin{aligned}
\hat{\rho}_{p}^{S}= & 2^{-1}\left(\mathbb{I}_{S}+p \hat{\sigma}_{3}+r_{1} \sqrt{1-p} \hat{\sigma}_{1}+r_{2} \sqrt{1-p} \hat{\sigma}_{2}\right. \\
& \left.\quad+r_{3}(1-p) \hat{\sigma}_{3}\right) \\
= & \frac{1}{2}\left(\mathbb{I}_{S}+\vec{r}_{p} \cdot \overrightarrow{\hat{\sigma}}\right),
\end{aligned}
$$

com o vetor de Bloch evoluído no tempo sendo

$$
\begin{aligned}
\vec{r}_{p} & =\left(r_{1}(p), r_{2}(p), r_{3}(p)\right) \\
& =\left(r_{1} \sqrt{1-p}, r_{2} \sqrt{1-p}, p+r_{3}(1-p)\right)(.59)
\end{aligned}
$$

Assim a CQ do átomo em um instante de tempo qualquer é dada pela Eq. 490 com $\left\{r_{j}\right\}_{j=1}^{2}$ substituídos por $\left\{r_{j}(p)\right\}_{j=1}^{2}$. Por conseguinte

$$
C\left(\hat{\rho}_{p}^{S}\right)=\sqrt{1-p} C\left(\hat{\rho}^{S}\right) .
$$

Ou seja, se $C\left(\hat{\rho}^{S}\right) \neq 0$ a $\mathrm{CQ}$ do átomo diminui monotonicamente indo a zero quando $t \rightarrow \infty$. A taxa de decaimento da $\mathrm{CQ}$ não depende do estado inicial, mas sim da taxa de decaimento do átomo; veja a Fig. 2 .

\section{Considerações Finais}

Como foi bem colocado por Robert Sppekens em 74, existem dois tipos de revolução em física. A primeira, a mais notada, é a aquela onde substituímos uma teoria antiga por outra teoria conceitualmente nova e mais abrangente, com a teoria da relatividade geral de Einstein e a mecânica quântica sendo importantes exemplos. O segundo tipo de revolução é mais lento, mas não menos importante, e envolve uma mudança de perspectiva sobre uma teoria existente. Um exemplo disso é o uso de princípios de simetria, de mínima ação e termodinâmicos em física. Tratando-se teorias físicas do ponto de vista de teoria de informação, ou seja, considerando-se estados físicos como sendo recursos, tem-se conseguido aperfeiçoar e generalizar esses princípios.

Com o desenvolvimento da ciência da informação quântica, mostrou-se que os mais variados aspectos de estados físicos (e.g., assimetria [63], atermalidade 61, emaranhamento 60, não localidade quântica [75], coerência quântica [69], discórdia quântica [65], etc) podem ser utilizados como recursos para a realização mais eficiente de diversos tipos de tarefas importantes para a ciência, tecnologia e sociedade. Nesse contexto, implementações no laboratório de protocolos que se utilizem desses recursos envolvem um balanço delicado entre o controle necessário do sistema quântico de interesse e da sua interação com o ambiente. Por isso é imprescindível levar em conta este último quando da descrição do sistema utilizado. Por conseguinte, é importante que, nas universidades e institutos de educação e pesquisa, trabalhemos antes e mais com a teoria de sistemas quânticos abertos.

Com a intenção de contribuir com esse projeto, neste artigo derivamos, de forma simples e livre de ambiguidades, a representação de Kraus para a dinâmica de sistemas quânticos abertos. Esperamos que esse trabalho ajude a desmistificar esse formalismo e estimule o seu uso, que se faz útil para descrever muitos sistemas físicos reais de interesse para várias áreas de pesquisa e em particular para CIQ. Nesse sentido, exemplificamos a aplicação desse formalismo estudando a dinâmica do estado e 


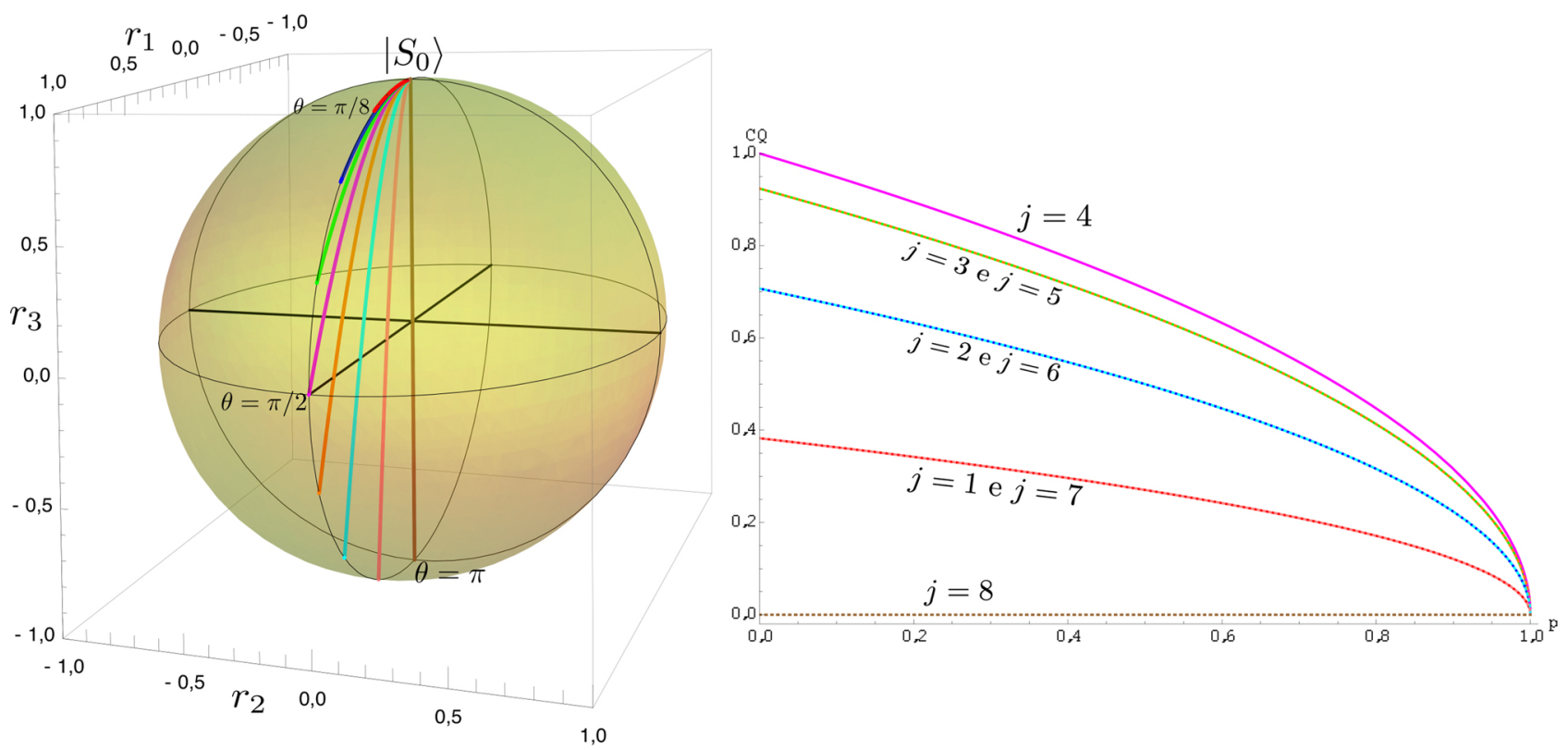

Figura 2: Qualquer estado $\hat{\rho}$ de um sistema de dois níveis pode ser representado por seu vetor de Bloch $\vec{r}$ em uma região de $\mathbb{R}^{3}$ conhecida como bola de Bloch. Em coordenadas esféricas, as componentes deste vetor são: $r_{1}=r \sin \theta \cos \phi$, $r_{2}=r \sin \theta \sin \phi$ e $r_{3}=r \cos \theta$, com $r \in[0,1], \theta \in[0, \pi]$ e $\phi \in[0,2 \pi)$. Nessa figura estão mostradas as evoluções temporais do vetor de Bloch (trajetórias no gráfico de cima) e da coerência quântica (gráfico de baixo) para estados iniciais puros ( $r=1$, estes estados estão na superfície da bola de Bloch, i.e., na esfera de Bloch) restritos ao plano $r_{2}=0$ $(\phi=0)$, com o ângulo $\theta=j \pi / 8(j=1, \cdots, 8)$ e evoluídos sob a ação do CAA. Estes estados iniciais são equivalentes a $\hat{\rho}=|\psi\rangle\langle\psi|\operatorname{com}| \psi\rangle=\cos (\theta / 2)\left|S_{0}\right\rangle+\sin (\theta / 2)\left|S_{1}\right\rangle$. No gráfico de cima obtemos os diferentes estados iniciais, e trajetórias, iniciando $\operatorname{com} \theta=\pi / 8$ (curva vermelha) e aumentando $\theta$ de $\pi / 8$ para obter o próximo estado mais abaixo. No gráfico de baixo as linhas pontilhadas são para $j>4$, e para $j \leq 4$ usamos linhas contínuas. Notamos que, para qualquer estado inicial, o estado assimptótico de $S$ é $\left|S_{0}\right\rangle$. Em todos os casos a coerência quântica diminui monotonicamente com o tempo parametrizado. No caso $j=8$ a $C Q$ é zero sempre. Observamos também que a $C Q$ é simétrica em relação ao ângulo $\theta=\pi / 2$, ou seja, $C\left(\hat{\rho}_{p}^{S}(\pi / 2+\delta)\right)=C\left(\hat{\rho}_{p}^{S}(\pi / 2-\delta)\right)$ para qualquer ângulo $\delta$ e tempo $p$. Isso indica a proporcionalidade entre a $C Q$ de um estado e a sua distância euclidiana até a linha incoerente $q\left|S_{0}\right\rangle\left\langle S_{0}|+(1-q)| S_{1}\right\rangle\left\langle S_{1}\right|$, com $0 \leq q \leq 1$.

da coerência quântica de um átomo de dois níveis interagindo com o vácuo do campo eletromagnético.

\section{Apêndices}

\section{A. Propriedades da dinâmica gerada}

Neste apêndice vamos verificar que os operadores de Kraus estão definidos em $\mathcal{H}_{S}$, que são lineares e que a dinâmica gerada é positiva e preserva o traço. Além disso, vamos discutir a não unicidade dos operadores de Kraus e o consequente limite no número de tais operadores que são necessários para descrever a dinâmica de $S$.

\section{A.1. Ação dos operadores de Kraus}

Vamos verificar que, como é esperado, os operadores de Kraus levam vetores de $\mathcal{H}_{S}$ em vetores de $\mathcal{H}_{S}$, o que se denota por $\hat{K}_{l}: \mathcal{H}_{S} \rightarrow \mathcal{H}_{S}$. Seja $|\xi\rangle$ um vetor qualquer de $\mathcal{H}_{S}$. Usando a resolução da unidade escrevemos

$$
\begin{aligned}
\hat{K}_{l}|\xi\rangle & =\mathbb{I}_{S} \hat{K}_{l} \mathbb{I}_{S}|\xi\rangle \\
& =\left(\sum_{j}\left|S_{j}\right\rangle\left\langle S_{j}\right|\right) \hat{K}_{l}\left(\sum_{k}\left|S_{k}\right\rangle\left\langle S_{k}\right|\right)|\xi\rangle \\
& =\sum_{j, k}\left\langle S_{j}\left|\hat{K}_{l}\right| S_{k}\right\rangle\left\langle S_{k} \mid \xi\right\rangle\left|S_{j}\right\rangle \\
& =\sum_{j, k}\left\langle S_{j} E_{l}|\hat{U}| S_{k} E_{0}\right\rangle\left\langle S_{k} \mid \xi\right\rangle\left|S_{j}\right\rangle \\
& =\sum_{j}\left\langle S_{j} E_{l}\left|\hat{U}\left(\sum_{k}\left|S_{k}\right\rangle\left\langle S_{k}\right|\right)\right| \xi\right\rangle \otimes\left|E_{0}\right\rangle\left|S_{j}\right\rangle \\
& =\sum_{j}\left\langle S_{j} E_{l}|\hat{U}| \xi E_{0}\right\rangle\left|S_{j}\right\rangle \\
& :=\sum_{j} c_{j \xi}^{(l)}\left|S_{j}\right\rangle,
\end{aligned}
$$


com os coeficientes complexos definidos como $c_{j \xi}^{(l)}:=$ $\left\langle S_{j} E_{l}|\hat{U}| \xi E_{0}\right\rangle$. Como qualquer combinação linear dos vetores de uma base de $\mathcal{H}_{S}$ também é um vetor de $\mathcal{H}_{S}$ vemos que $\hat{K}_{l}|\xi\rangle \in \mathcal{H}_{S}$ e por conseguinte de fato $\hat{K}_{l}: \mathcal{H}_{S} \rightarrow \mathcal{H}_{S}$ para qualquer valor de $l$.

De forma similar, podemos escrever $\hat{K}_{l}^{\dagger}|\xi\rangle=$ $\sum_{j} d_{j \xi}^{(l)}\left|S_{j}\right\rangle$, com $d_{j \xi}^{(l)}=\left\langle S_{j} E_{0}\left|\hat{U}^{\dagger}\right| \xi E_{l}\right\rangle$, para ver que $\hat{K}_{l}^{\dagger}|\xi\rangle \in \mathcal{H}_{S} \forall l$. Esse fato será útil e.g. para a subsequente análise da positividade da dinâmica gerada.

\section{A.2. Linearidade dos operadores de Kraus}

Consideremos uma combinação linear de um conjunto qualquer de vetores $\left|\zeta_{m}\right\rangle \in \mathcal{H}_{S}$ (não necessariamente ortogonais), $|\xi\rangle:=\sum_{m} c_{m}\left|\zeta_{m}\right\rangle$ com $c_{m} \in \mathbb{C}$. Um operador $\hat{L}: \mathcal{H}_{S} \rightarrow \mathcal{H}_{S}$ é dito linear se $\hat{L}\left(\sum_{m} c_{m}\left|\zeta_{m}\right\rangle\right)=\sum_{m} c_{m} \hat{L}\left|\zeta_{m}\right\rangle$. Para os operadores de Kraus, podemos utilizar a Eq. (61), e a linearidade de $\hat{U}$ e do produto interno, para escrever

$$
\begin{aligned}
\hat{K}_{l}\left(\sum_{m} c_{m}\left|\zeta_{m}\right\rangle\right) & =\hat{K}_{l}|\xi\rangle \\
& =\sum_{j}\left\langle S_{j} E_{l}|\hat{U}| \xi E_{0}\right\rangle\left|S_{j}\right\rangle \\
& =\sum_{j}\left\langle S_{j} E_{l}\left|\hat{U} \sum_{m} c_{m}\right| \zeta_{m}\right\rangle \otimes\left|E_{0}\right\rangle\left|S_{j}\right\rangle \\
& =\sum_{j}\left\langle S_{j} E_{l}\left|\hat{U} \sum_{m} c_{m}\right| \zeta_{m} E_{i}\right\rangle\left|S_{j}\right\rangle \\
& =\sum_{m} c_{m} \sum_{j}\left\langle S_{j} E_{l}|\hat{U}| \zeta_{m} E_{i}\right\rangle\left|S_{j}\right\rangle \\
& =\sum_{m} c_{m} \hat{K}_{l}\left|\zeta_{m}\right\rangle .
\end{aligned}
$$

De forma similar pode-se mostrar que $\hat{K}_{l}^{\dagger}\left(\sum_{m} c_{m}\right.$ $\left.\left|\zeta_{m}\right\rangle\right)=\sum_{m} c_{m} \hat{K}_{l}^{\dagger}\left|\zeta_{m}\right\rangle$. Em suma, nestas duas primeiras sub-seções deste Apêndice mostramos que os operadores de Kraus $\hat{K}_{l}$, e $\hat{K}_{l}^{\dagger}$, são operadores lineares e que estão definidos em $\mathcal{H}_{S}$.

\section{A.3. A dinâmica gerada preserva positivi- dade}

Vamos mostrar que $\hat{\rho}_{t}^{S}$ é positivo semi-definido. Para tal, comecemos considerando um vetor qualquer $|\xi\rangle \in \mathcal{H}_{S}$, para o qual, pela linearidade do produto matricial,

$$
\left\langle\xi\left|\hat{\rho}_{t}^{S}\right| \xi\right\rangle=\sum_{l}\left\langle\xi\left|\hat{K}_{l} \hat{\rho}^{S} \hat{K}_{l}^{\dagger}\right| \xi\right\rangle
$$

Mostramos acima que $\hat{K}_{l}^{\dagger}: \mathcal{H}_{S} \rightarrow \mathcal{H}_{S}$. Usemos essa propriedade aqui para definir $\hat{K}_{l}^{\dagger}|\xi\rangle=:\left|\xi_{l}\right\rangle \in \mathcal{H}_{S}$, que implica em $\left\langle\xi_{l}|=| \xi_{l}\right\rangle^{\dagger}=\langle\xi| \hat{K}_{l}$, e assim escrever

$$
\begin{aligned}
\left\langle\xi\left|\hat{\rho}_{t}^{S}\right| \xi\right\rangle & =\sum_{l} \underbrace{\left\langle\xi_{l}\left|\hat{\rho}^{S}\right| \xi_{l}\right\rangle}_{\geq 0} \\
& \geq 0 .
\end{aligned}
$$

Na Eq. (64) utilizamos a positividade do operador densidade inicial de $S$.

\section{A.4. A dinâmica gerada preserva o traço}

Além da positividade demonstrada anteriormente, para que uma distribuição de probabilidades válida seja gerada, o operador densidade deve ter traço igual a um para todos os instantes de tempo $t$. Temos que

$$
\begin{aligned}
\operatorname{Tr}\left(\hat{\rho}_{t}^{S}\right) & =\operatorname{Tr}\left(\sum_{l} \hat{K}_{l} \hat{\rho}^{S} \hat{K}_{l}^{\dagger}\right) \\
& =\sum_{l} \operatorname{Tr}\left(\hat{K}_{l} \hat{\rho}^{S} \hat{K}_{l}^{\dagger}\right) \\
& =\sum_{l} \operatorname{Tr}\left(\hat{K}_{l}^{\dagger} \hat{K}_{l} \hat{\rho}^{S}\right) \\
& =\operatorname{Tr}\left(\left(\sum_{l} \hat{K}_{l}^{\dagger} \hat{K}_{l}\right) \hat{\rho}^{S}\right) .
\end{aligned}
$$

$\mathrm{Na}$ última equação utilizamos a linearidade da função traço e a sua propriedade cíclica. Agora usemos a resolução da unidade para ver que

$$
\begin{aligned}
& \sum_{l} \hat{K}_{l}^{\dagger} \hat{K}_{l}=\sum_{l} \mathbb{I}_{S} \hat{K}_{l}^{\dagger} \mathbb{I}_{S} \hat{K}_{l} \mathbb{I}_{S} \\
& =\sum_{l m n o}\left|S_{m}\right\rangle\left\langle S_{m}\left|\hat{K}_{l}^{\dagger}\right| S_{n}\right\rangle\left\langle S_{n}\left|\hat{K}_{l}\right| S_{o}\right\rangle\left\langle S_{o}\right| \\
& =\sum_{l m n o}\left|S_{m}\right\rangle\left\langle S_{m} E_{0}\left|\hat{U}^{\dagger}\right| S_{n} E_{l}\right\rangle\left\langle S_{n} E_{l}|\hat{U}| S_{o} E_{0}\right\rangle\left\langle S_{o}\right| \\
& =\sum_{m o}\left|S_{m}\right\rangle\left\langle S_{m} E_{0}\left|\hat{U}^{\dagger} \sum_{n l}\right| S_{n} E_{l}\right\rangle\left\langle S_{n} E_{l}|\hat{U}| S_{o} E_{0}\right\rangle\left\langle S_{o}\right| \\
& =\sum_{m o}\left|S_{m}\right\rangle\left\langle S_{m} E_{0}\left|\hat{U}^{\dagger} \hat{U}\right| S_{o} E_{0}\right\rangle\left\langle S_{o}\right| \\
& =\sum_{m o}\left|S_{m}\right\rangle\left\langle S_{m} E_{0} \mid S_{o} E_{0}\right\rangle\left\langle S_{o}\right| \\
& =\sum_{m o}\left|S_{m}\right\rangle \delta_{m o}\left\langle S_{o}\right| \\
& =\sum_{m}\left|S_{m}\right\rangle\left\langle S_{m}\right| \\
& =\mathbb{I}_{S} .
\end{aligned}
$$

Esta igualdade garante ${ }^{6}$ que a dinâmica descrita acima preserva o traço do operador densidade evoluído do sistema, i.e., $\operatorname{Tr}\left(\hat{\rho}_{t}^{S}\right)=\operatorname{Tr}\left(\hat{\rho}^{S}\right)=1$, e portanto que gera um estado quântico válido para qualquer instante de tempo.

\footnotetext{
${ }^{6}$ Vale observar que, em contraste como o formalismo geral de operações quânticas, no contexto da dinâmica sistemaambiente considerada aqui $\sum_{l} \hat{K}_{l}^{\dagger} \hat{K}_{l}=\mathbb{I}_{S}$ é um fato e não um condição a ser satisfeita pelos operadores de Kraus para garantir que a dinâmica gerada preserve o traço.
} 


\section{A.5. Não unicidade dos operadores de Kraus}

O conjunto de operadores de Kraus que gera um mapa $\$: \hat{\rho}^{S} \rightarrow \hat{\rho}_{t}^{S}$ não é único. Para verificar essa afirmação consideremos [24]:

$$
\hat{K}_{l}^{\prime}:=\sum_{n} V_{l n} \hat{K}_{n}
$$

com $V_{l n}$ sendo os elementos de matriz de um operador unitário qualquer $\left(\hat{V}^{\dagger} \hat{V}=\mathbb{I}\right)$. O estado de $S$ obtido usando-se os operadores $\hat{K}_{l}^{\prime}$ é:

$$
\begin{aligned}
\sum_{l} \hat{K}_{l}^{\prime} \hat{\rho}^{S} \hat{K}_{l}^{\prime \dagger} & =\sum_{l}\left(\sum_{n} V_{l n} \hat{K}_{n}\right) \hat{\rho}^{S}\left(\sum_{m} V_{l m}^{*} \hat{K}_{m}^{\dagger}\right) \\
& =\sum_{n m} \sum_{l} V_{l m}^{*} V_{l n} \hat{K}_{n} \hat{\rho}^{S} \hat{K}_{m}^{\dagger} \\
& =\sum_{n} \hat{K}_{n} \hat{\rho}^{S} \hat{K}_{n}^{\dagger} \\
& =\hat{\rho}_{t}^{S}
\end{aligned}
$$

pois

$$
\sum_{l} V_{l m}^{*} V_{l n}=\sum_{l}\left(\hat{V}^{\dagger}\right)_{m l} V_{l n}=\left(\hat{V}^{\dagger} \hat{V}\right)_{m n}=\delta_{m n} .
$$

Ou seja, os conjuntos de operadores $\left\{\hat{K}_{l}^{\prime}\right\}$ e $\left\{\hat{K}_{l}\right\}$ geram a mesma dinâmica quântica para $S$.

No contexto da dinâmica sistema-ambiente que estamos interessados aqui, uma relação como a dada na Eq. (67) é obtida se, depois de o sistema e ambiente evoluírem sob a ação de $\hat{U}$, uma transformação unitária $\hat{V}$ for aplicada ao ambiente. Assim, a composição

$$
\hat{U}^{\prime}=\left(\mathbb{I}_{S} \otimes \hat{V}\right) \hat{U}
$$

nos leva aos operadores de Kraus:

$$
\begin{aligned}
& \left\langle S_{k}\left|\hat{K}_{l}^{\prime}\right| S_{m}\right\rangle=\left\langle S_{k} E_{l}\left|\hat{U}^{\prime}\right| S_{m} E_{0}\right\rangle \\
& =\left\langle S_{k} E_{l}\left|\left(\mathbb{I}_{S} \otimes \hat{V}\right) \hat{U}\right| S_{m} E_{0}\right\rangle \\
& =\left\langle S_{k} E_{l}\left|\left(\mathbb{I}_{S} \otimes \hat{V}\right)\left(\mathbb{I}_{S} \otimes \sum_{n}\left|E_{n}\right\rangle\left\langle E_{n}\right|\right) \hat{U}\right| S_{m} E_{0}\right\rangle \\
& =\sum_{n}\left(\left\langle S_{k}\left|\otimes\left\langle E_{l}|\hat{V}| E_{n}\right\rangle\left\langle E_{n}\right|\right) \hat{U} \mid S_{m} E_{0}\right\rangle\right. \\
& =\sum_{n} V_{l n}\left\langle S_{k} E_{n}|\hat{U}| S_{m} E_{0}\right\rangle \\
& =\sum_{n} V_{l n}\left\langle S_{k}\left|\hat{K}_{n}\right| S_{m}\right\rangle,
\end{aligned}
$$

que é equivalente à Eq. 67). Vale observar que essas rotações locais, aplicadas depois da dinâmica conjunta, raramente têm alguma implicação relevante nas funções que consideramos em ciência da informação quântica. Ou seja, neste contexto podemos, em geral, desconsiderar a não unicidade dos operadores de Kraus.

\section{A.6. Limite no número de operadores de Kraus necessários para descrever uma dinâmica quântica}

Como a dimensão do ambiente (e de $\hat{U}$ ) não é limitada, não há um motivo aparente para esperarmos que o número de operadores de Kraus deva ser limitado. Não obstante, vamos mostrar que um conjunto de até $d_{S}^{2}$ operadores de Kraus é suficiente para gerar qualquer dinâmica de um sistema com dimensão $d_{S}$.

Os operadores de Kraus estão definidos em $\mathcal{H}_{S}$ (i.e., $\hat{K}_{l}: \mathcal{H}_{S} \rightarrow \mathcal{H}_{S}$ ) e, por conseguinte, podem ser representados por matrizes complexas com dimensão $d_{S} \mathrm{x} d_{S}$. O espaço formado por essas matrizes é denotado por $\mathbb{C}^{d_{S} \times d_{S}}$. Seguindo a Ref. [24], vamos começar mostrando que para a matriz Hermitiana $\hat{W}$ definida por

$$
W_{l m}:=\operatorname{Tr}\left(\hat{K}_{l}^{\dagger} \hat{K}_{m}\right)
$$

temos que $\operatorname{rank}(\hat{W}) \leq d_{S}^{2}$, em que o rank de $\hat{W}$ é definido como o número de seus vetores coluna que são linearmente independentes (LI) [76]. Para isso, notemos que existe uma base ortonormal para $\mathbb{C}^{d_{S} \times d_{S}}$ com $d_{S}^{2}$ elementos, o que implica que não mais do que $d_{S}^{2}$ dos operadores $\hat{K}_{l}$ podem ser LI. Suponhamos, sem perda de generalidade, que os primeiros $d_{S}^{2}$ operadores $\hat{K}_{l}$ são LI. Com isso podemos escrever, para $m>d_{S}^{2}$, a combinação linear:

$$
\hat{K}_{m}=\sum_{l=1}^{d_{S}^{2}} c_{l}^{(m)} \hat{K}_{l}
$$

$\operatorname{com} c_{l}^{(m)} \in \mathbb{C}$. Pode-se verificar facilmente que isso implica que o $m$-ésimo vetor coluna de $\hat{W}$, para $m>d_{S}^{2}$, é uma combinação linear $\operatorname{dos} d_{S}^{2}$ primeiros vetores coluna:

$$
W_{l m}=\sum_{j=1}^{d_{S}^{2}} c_{j}^{(m)} W_{l j}
$$

Fica provado assim que

$$
\operatorname{rank}(\hat{W}) \leq d_{S}^{2}
$$

Utilizando esse resultado e o fato de que $\hat{W}$ é uma matriz Hermitiana, podemos diagonalizá-la via uma transformação unitária $\hat{V}$ :

$$
\hat{W}^{\prime}:=\hat{V} \hat{W} \hat{V}^{\dagger}
$$

Revista Brasileira de Ensino de Física, vol. 38, nº 2, e2307, 2016 
Os $d_{S}^{2}$ elementos diagonais possivelmente não nulos de $\hat{W}^{\prime}$ são obtidos como segue:

$$
\begin{aligned}
W_{j j}^{\prime} & =\left(\hat{V} \hat{W} \hat{V}^{\dagger}\right)_{j j}=\sum_{l} V_{j l}\left(\hat{W} \hat{V}^{\dagger}\right)_{l j} \\
& =\sum_{l m} V_{j l} W_{l m}\left(\hat{V}^{\dagger}\right)_{m j}=\sum_{l m} V_{j l} \operatorname{Tr}\left(\hat{K}_{l}^{\dagger} \hat{K}_{m}\right) V_{j m}^{*} \\
& =\operatorname{Tr} \sum_{l} V_{j l} \hat{K}_{l}^{\dagger} \sum_{m} V_{j m}^{*} \hat{K}_{m}=\operatorname{Tr}\left(\hat{K}_{j}^{\prime \dagger} \hat{K}_{j}^{\prime}\right) \cdot(75)
\end{aligned}
$$

Temos assim um conjunto com até $d_{S}^{2}$ operadores de Kraus $\hat{K}_{j}^{\prime}=\sum_{m} V_{j m}^{*} \hat{K}_{m}$, que geram a mesma dinâmica que o conjunto $\hat{K}_{m}$.

\section{Agradecimentos}

Este trabalho foi realizado com o suporte financeiro do CNPq, processos 441875/2014-9 e 303496/2014-2, do Instituto Nacional de Ciência e Tecnologia de Informação Quântica, processo 2008/57856-6, e da CAPES, processo 6531/2014-08. Agradeço a hospitalidade do Instituto de Física e do Grupo de Espectroscopia Laser da Universidad de la República, Uruguai, onde este artigo foi completado, e a Adriana Auyuanet por sugestões e por discussões sobre o tema deste artigo. Agradeço também aos Revisores por suas sugestões e críticas, que ajudaram a melhorar este artigo.

\section{Referências}

[1] J. Kakalios, The Amazing Story of Quantum Mechanics (Gotham Books, Nova York, 2010).

[2] IOP, The Age of the Qubit: A New Era of Quantum Information in Science and Technology, disponível em http://www.iop.org/publications/ iop/2011/file_52078.pdf.

[3] R. Laflamme, Embrace the Quantum Revolution, disponível em https://www.youtube.com/watch? $\mathrm{v}=\mathrm{pEJuowHK} 2 \mathrm{uA}$.

[4] R. de Lima Rodrigues, Rev. Bras. Ensino Fís. 19, 68 (1997).

[5] B.L. Van der Waerden, Sources of Quantum Mechanics (Dover Publications, Nova York, 1967).

[6] T.D. Ladd, F. Jelezko, R. Laflamme, Y. Nakamura, C. Monroe and J.L. O'Brien, Nature 464, 45 (2010).

[7] I.M. Georgescu, S. Ashhab and F. Nori, Rev. Mod. Phys. 86, 153 (2014).

[8] A. Ekert and R. Renner, Nature 507, 443 (2014).

[9] V. Giovannetti, S. Lloyd and L. Maccone, Nat. Photon. 5, 222 (2011).

[10] B.M. Escher, R.L. de Matos Filho e L. Davidovich, Braz. J. Phys. 41, 229 (2011).

[11] C. Jarzynski, Nat. Phys. 11, 105 (2015).
[12] J. McFadden and J. Al-Khalili, Life on the Edge: The Coming of Age of Quantum Biology (Crown Publishers, Nova York, 2014).

[13] M. Schulda, I. Sinayskiya and F. Petruccione, Contemp. Phys. 56, 172 (2015).

[14] M. Schuld, I. Sinayskiy and F. Petruccione, arXiv:1408.7005.

[15] N. Wiebe, A. Kapoor and K.M. Svore, arXiv:1412.3489.

[16] S.E. Venegas-Andraca, Quantum Inf. Process. 14, 1535 (2015).

[17] Caltech Quantum Frontiers, Blog Post: Can a game teach kids quantum mechanics?, disponível em http://quantumfrontiers.com/2013/10/17/ can-a-game-teach-kids-quantum-mechanics/

[18] R. Müller and H. Wiesner, Am. J. Phys. 70, 200 (2002).

[19] I.M. Greca, M.A. Moreira e V.E. Herscovitz, Rev. Bras. Ensino Fís 23, 444 (2001).

[20] S.B. McKagan, K.K. Perkins, M. Dubson, C. Malley, S. Reid, R. LeMaster and C.E. Wieman, Am. J. Phys. 76, 406 (2008).

[21] M. Tomandl, T. Mieling, C.M.L.-V. Kroon, M. Hopf and M. Arndt, Sci. Rep. 5, 14108 (2015).

[22] University of Bristol, Quantum in the Cloud, disponível em http://www.bristol.ac.uk/physics/ research/quantum\/outreach/qcloud/.

[23] W.H. Zurek, Phys. Today 67, 44 (2014).

[24] M.A. Nielsen and I.L. Chuang, Quantum Computation and Quantum Information (Cambridge University Press, Cambridge, 2000).

[25] J. Preskill, Quantum Information and Computation, disponível em http://theory.caltech.edu/ people/preskill/ph229/.

[26] M.M. Wilde, Quantum Information Theory (Cambridge University Press, Cambridge, 2013).

[27] J. Maziero, Rev. Bras. Ensino Fís. 37, 1314 (2015).

[28] K. Kraus, Ann. Phys. 64, 311 (1971).

[29] K. Kraus, States, Effects, and Operations: Fundamental Notions of Quantum Theory (Springer, Berlin, 1983).

[30] C.A. Brasil, F.F. Fanchini e R. de Jesus Napolitano, Rev. Bras. Ensino Fís. 35, 1303 (2013).

[31] G.A. Prataviera e S.S. Mizrahi, Rev. Bras. Ensino Fís. 36, 4303 (2014).

[32] T.B.L. Kist, M. Orszag, T.A. Brun and L. Davidovich, J. Opt. B: Quantum Semiclass. Opt. 1, 251 (1999).

[33] D.O. Soares-Pinto, M.H.Y. Moussa, J. Maziero, E.R. deAzevedo, T.J. Bonagamba, R.M. Serra and L.C. Céleri, Phys. Rev. A 83, 062336 (2011).

[34] P. Pechukas, Phys. Rev. Lett. 73, 1060 (1993).

[35] R. Alicki, Phys. Rev. Lett. 75, 3020 (1995).

[36] A. Shaji and E.C.G. Sudarshan, Phys. Lett. A 341, 48 (2005). 
[37] C.A. Rodríguez-Rosario, K. Modi, A.-m. Kuah, A. Shaji and E.C.G. Sudarshan, J. Phys. A: Math. Theor. 41, 205301 (2008).

[38] A. Shabani and D.A. Lidar, Phys. Rev. Lett. 102, 100402 (2009).

[39] A. Brodutch, A. Datta, K. Modi, Á. Rivas e C.A. Rodríguez-Rosario, Phys. Rev. A 87, 042301 (2013).

[40] F. Buscemi, Phys. Rev. Lett. 113, 140502 (2014).

[41] J. Maziero and F.M. Zimmer, Phys. Rev. A 86, 042121 (2012)

[42] O. Jiménez Farías, G.H. Aguilar, A. ValdésHernández, P.H. Souto Ribeiro, L. Davidovich and S.P. Walborn, Phys. Rev. Lett. 109, 150403 (2012).

[43] M. Schlosshauer, Decoherence and the Quantum-toClassical Transition (Springer, Berlin, 2007).

[44] W.H. Zurek, Nat. Phys. 5, 181 (2009).

[45] T.F. Gallagher, Rydberg Atoms (Cambridge University Press, Nova York, 1994).

[46] J.A.C. Gallas, Cad. Cat. Ens. Fis. 3, 41 (1986).

[47] R. Ejnisman e P. Nussenzveig, Rev. Bras. Ensino Fís. 19, 90 (1997).

[48] R.V. Noorden, Nature 498, 22 (2013).

[49] M.A. Kastner, Phys. Today 46, 24 (1993).

[50] P. Recher and B. Trauzettel, Nanotech. 21, 302001 (2010).

[51] J.Q. You and F. Nori, Nature 474, 589 (2011).

[52] R.J. Glauber, Rev. Mod. Phys. 78, 1267 (2006).

[53] G.S. Agarwal, Quantum Statistical Theories of Spontaneous Emission (Springer, Berlin, 1974).

[54] J. Maziero, R. Auccaise, L.C. Celeri, D.O. SoaresPinto, E.R. deAzevedo, T.J. Bonagamba, R.S. Sarthour, I.S. Oliveira e R.M. Serra, Braz. J. Phys. 43, 86 (2013).

[55] R.P. Feynman, R.B. Leighton and M. Sands, The Feynman Lectures on Physics, Volume 3 (AddisonWesley Publishing Company, Massachusetts, 1965).

[56] L. Mandel and E. Wolf, Optical Coherence and Quantum Optics (Cambridge University Press, Nova York, 1995).

[57] C.-M. Li, N. Lambert, Y.-N. Chen, G.-Y. Chen and F. Nori, Sci. Rep. 2, 885 (2012).

[58] I. Devetak, A.W. Harrow and A. Winter, IEEE Trans. Inf. Th. 54, 4587 (2008).

[59] G. Gour and R.W. Spekkens, New J. Phys. 10, 033023 (2008).

[60] R. Horodecki, P. Horodecki, M. Horodecki and K. Horodecki, Rev. Mod. Phys. 81, 865 (2009).

[61] F.G.S.L. Brandão, M. Horodecki, J. Oppenheim, J.M. Renes and R.W. Spekkens, Phys. Rev. Lett. 111, 250404 (2013).

[62] V. Veitch, S.A.H. Mousavian, D. Gottesman and J. Emerson, New J. Phys. 16, 013009 (2014).

[63] I. Marvian and R.W. Spekkens, Nat. Comm. 5, 3821 (2014).

[64] F.G.S.L. Brandão and G. Gour, Phys. Rev. Lett. 115, 070503 (2015).
[65] M. Horodecki and J. Oppenheim, Int. J. Mod. Phys. B 27, 1345019 (2013).

[66] J. Åberg, Phys. Rev. Lett. 113, 150402 (2014).

[67] T. Baumgratz, M. Cramer and M.B. Plenio, Phys. Rev. Lett. 113, 140401 (2014).

[68] F. Levi and F. Mintert, New J. Phys. 16, 033007 (2014).

[69] A. Winter and D. Yang, arXiv:1506.07975.

[70] A. Streltsov, U. Singh, H.S. Dhar, M.N. Bera and G. Adesso, Phys. Rev. Lett. 115, 020403 (2015).

[71] M. Lostaglio, K. Korzekwa, D. Jennings and T. Rudolph, Phys. Rev. X 5, 021001 (2015).

[72] Z. Xi, Y. Li and H. Fan, Sci. Rep. 5, 10922 (2015).

[73] D.P. Pires, L.C. Céleri and D.O. Soares-Pinto, Phys. Rev. A 91, 042330 (2015).

[74] R.W. Spekkens, The Invasion of Physics by Information Theory, Perimeter Institute Recorded Seminar Archive, disponível em http://pirsa.org/ displayFlash.php?id=14030085

[75] J.I. de Vicente, J. Phys. A: Math. Theor. 47, 424017 (2014).

[76] R.A. Horn and C.R. Johnson, Matrix Analysis (Cambridge University Press, Nova York, 2013). 